\title{
SOME EFFECTS OF SIZE ON JUDGMENTS OF WEIGHT.
}

BY DR. H. K. WOLFE.

Sight may well be called the universal sense. It contributes, perhaps, as few of the original elements of our knowledge as either touch or hearing, and in this regard it should not rank much superior to some of the other senses. On the other hand, its facility of association is so marked that psychologists of all times have been unable to agree upon the line separating ' native' and 'acquired' powers. Even in the new psychology there is little unanimity on the degree of 'voluminousness' possessed by sensations from the retina alone. One point, however, has been made remarkably clear by the newer investigations of the senses, viz. : the complexity of even the so-called simple perceptions.

A very crude analysis reveals the small part actually taken by retinal impressions in our knowledge of the external world. And yet our eyes are so actively engaged in the search for knowledge, that scarcely any phase or degree of the stimuli which act on other senses fails to produce also some effect on the retina. While this fact is especially noticeable in connection with touch and the muscular sense, it may also be observed in hearing, and even in case of the lower senses, taste and smell. Sight is indeed becoming so nearly a mirror of the senses that we often forget that its value depends largely upon its reflective power. Its fine shades of discrimination and its wonderfully tenacious associative activity enable it to become a factor in nearly all our perceptions, not only without effort but with a naturalness resembling that of instinct itself.

We are momentarily reminded of the versatility of sight. Its suggestibility seems unlimited. The taste of a strawberry, the smell of a rose, the feeling of a file, injuries and blood, the movements of musicians-in all these instances sight seems to 
stimulate the appropriate sense organ, and to some extent it acts as a substitute sense. The degree of substitution, or indeed the existence of any such action at all, depends, of course, upon the previous action of the proper sense in connection with sight.

Whether the sense of sight is assuming the functions of the other senses to their detriment is, perhaps, uncertain. The relative importance of the senses is undoubtedly changing, and in this way sight is certainly distancing all others. Unless our power of acquiring knowledge is also increasing, it follows that the other senses must lose. It is not inconceivable that sight may yet develop much further, and that we shall succeed in breaking up the real or supposed circuit from the written word through hearing to the centers of speech and understanding. Why should we not obtain as clear an idea of the contents of a written page from one glance as of a picture? In a future paper I hope to consider some pedagogical questions illuminated by recent investigations along this line. At present we are concerned with one or two specific phases of the influence of sight on other sensations.

The basis for this article is a series of experiments made during the past four years, chiefly on advanced students of the University of Nebraska. The work was suggested by the wellknown difficulty of judging the weight of light bodies in terms of pounds, or of heavy bodies. A pound of lead was known to be psychologically heavier than a pound of feathers; but I wish to know as nearly as possible in the time at my disposal, how much heavier, or how much feathers is as heavy as a pound of lead. This latter form of the question suggests the only method that can be employed with any degree of accuracy. It is, moreover, the only method recognized in experimental psychology for quantitative work.

I knew of no attempts along this line having been made or proposed, and, as is always to be expected in such cases, the first results were surprising and instructive. ${ }^{2}$ Wood and lead

'In the summer of 1894 , after my experiments for the first year had been made, Dr. F. B. Dresslar published in the American Journal of Psychology, Vol. VI., page $3^{13}$ ff., an article on 'The Psychology of Touch,' in which this same problem was attacked in a different manner. Dr. Dresslar experimented by proxy chiefly on school children, who were requested to arrange in order of 
were selected as the best materials at our command. Ten disks of wood three-quarters of an inch thick and varying regularly in diameter from two to twelve inches were prepared. These were well seasoned and were numbered in order from one to ten (I.-X.), beginning with the smallest. These ten disks served throughout all experiments hereinafter described.

The corresponding series of lead weights was not so soon determined. I first prepared fifteen lead disks, making the lightest about half as heavy as the smallest wood disk. Some preliminary experiments soon proved that lighter lead weights would be necessary. Three smaller weights were added, the lightest weighing a little less than one-fourth as much as the smallest wood disk. The experiments of the first year were made with these eighteen lead weights. It was found that the lead weights were too few. For the second year another series was made, beginning with one-seventh the weight of the smallest wood disk, and ending with number twenty-seven, which

weight a series of brass cylinders having the same diameter but varying in length by half inches, from one and a half inches to five inches. The cylinders were, in fact, of equal weight, but appeared unequal on account of the difference in size.

The next year Dr. Seashore published in Studies From the Yale Psychological Laboratory, Vol. III., a very interesting paper on 'Measurements of Illusions and Hallucinations in Normal Life,' in which he presents another method and its results. Dr. Dresslar did not emphasize the quantitative relations, since his method was not adapted to quantitative determinations. It is also a question whether the method employed by Dr. Seashore will yield true quantitative results. His standards were all of one size and differed in weight, while the companion series were all of one weight and differed in size. At first thought he would seem thus to avoid the double illusion. But when the smallest weight is compared with the lightest, or when the largest weight is compared with the heaviest, there is evidently a double illusion very unlike that obtained by comparing the medium size with the medium weight. Something of the nature of the illusion is also suggested by the conditions of the experiment. Suspicion is aroused by a series of cylinders uniform in size but varying in weight. With trained observers such as Dr. Seashore employed there is, of course, little objection on this account. For the untrained observer such as I have used, my own series seem to furnish the least objectionable method. It is the natural process and each person uses it almost daily. Before a quantitative relation between size and weight could be established, my method would require experiments on several substances. But it is doubtful whether such a relation can be established in any other way, so as to represent the normal, unconscious effect of size on our judgments of weight. 
weighed slightly more than the largest wood disk. The same series was used also during the third and fourth years. It was impossible to prepare lead balls for this work and equally difficult to secure cylinders or disks of uniform diameter. Lead disks of four diameters were, therefore, used ( $1 / 2$ in., I in., I I $/ 2$ in., 2 in.). While this very considerable change in diameter introduces a cause of error which must be regretted and which will be referred to later on, it seemed to me the best solution practicable at the time.

The process of judging was the same every year. For this kind of work I do not believe it best to restrict the method of students. Constant method seems to be less important than constant subject. Each student lifted the weights as he pleased, with one hand or two hands, by edges, on tips of fingers, or in any way that suggested itself to the individual. Two to four students worked at the same time (on different parts of the series of course), and under circumstances which prevented any assistance whatever, except perhaps in the suggestion of variations in method of holding the weights. Either myself or an assistant was always present. Usually about twenty minutes were required for a series.

It would be interesting to have before us the separate judgments of each student, but with so large a number of individuals this is, perhaps, impracticable. I, therefore, have arranged the members of each class in alphabetical order, men and women in separate lists. Groups, $a, b, c$, etc., are then formed, consisting of approximately ten students each, in no case interrupting the alphabetical order. The tables contain the averages of each group for the various wood weights, together with the average variation of the individuals from the average of their group.

The results from the individuals of the first group of ten men, and also from the first group of ten women for the year 1896 are given in Table $I$. The figures at the head of the table show the weight of the wood disks, other numbers show the weight of the lead. These results are fairly characteristic of the entire company examined. They show the astonishing differences between the judgments of the sexes very well. They 
do not happen to contain the extreme judgments, but they have nearly as large variations as the other groups, as may be observed in Table II. While a more or less regular progression from light to heavy is generally to be observed, this is sometimes seen to be inexplicably slow, as in numbers 5 and Io of the women, and 8 and 9 of the men. The irregularity of number 9 of the men is an exceptional case.

TABLE I.

Showing individual judgments of men and women.

\begin{tabular}{|c|c|c|c|c|c|c|c|c|c|c|}
\hline Wood. & 15.5 & 35 & $6 I$ & 87.5 & 126.5 & 174 & $23 I$ & 295 & 364 & 525 \\
\hline \multicolumn{11}{|l|}{ Men. } \\
\hline $\begin{array}{r}1 \\
2 \\
3 \\
4 \\
5 \\
6 \\
7 \\
8 \\
9 \\
10\end{array}$ & $\begin{array}{l}7.5 \\
6.0 \\
7.5 \\
6.0 \\
3.8 \\
3.8 \\
3.8 \\
3.8 \\
6.0 \\
6.0\end{array}$ & $\begin{array}{r}11.2 \\
11.2 \\
23.0 \\
2.2 \\
11.2 \\
9.3 \\
7.5 \\
6.0 \\
6.0 \\
7.5\end{array}$ & $\begin{array}{r}27.0 \\
13.5 \\
27.0 \\
23.0 \\
200 \\
13.5 \\
13.5 \\
7.5 \\
11.2 \\
13.5\end{array}$ & $\begin{array}{r}34.0 \\
17.5 \\
34.0 \\
34.0 \\
27.0 \\
17.5 \\
20.0 \\
9.3 \\
13.5 \\
20.0\end{array}$ & $\begin{array}{l}70.0 \\
27.0 \\
50.0 \\
70.0 \\
50.0 \\
23.0 \\
27.0 \\
17.5 \\
17.5 \\
23.0\end{array}$ & $\begin{array}{r}84 \\
39 \\
70 \\
109 \\
84 \\
39 \\
39 \\
20 \\
20 \\
27\end{array}$ & $\begin{array}{r}109 \\
70 \\
84 \\
147 \\
109 \\
50 \\
50 \\
23 \\
34 \\
34\end{array}$ & $\begin{array}{r}147 \\
109 \\
147 \\
204 \\
147 \\
70 \\
84 \\
34 \\
20 \\
50\end{array}$ & $\begin{array}{r}204 \\
198 \\
204 \\
231 \\
204 \\
109 \\
147 \\
39 \\
27 \\
109\end{array}$ & $\begin{array}{r}274 \\
274 \\
274 \\
274 \\
231 \\
147 \\
231 \\
50 \\
39 \\
204\end{array}$ \\
\hline \multicolumn{11}{|l|}{ Women. } \\
\hline $\begin{array}{r}1 \\
2 \\
3 \\
4 \\
5 \\
6 \\
7 \\
8 \\
9 \\
10\end{array}$ & $\begin{array}{l}3.8 \\
2.2 \\
3.8 \\
2.2 \\
3.8 \\
3.8 \\
1.7 \\
3.8 \\
2.2 \\
1.7\end{array}$ & $\begin{array}{r}6.0 \\
3.8 \\
7.5 \\
6.0 \\
6.0 \\
11.2 \\
2.2 \\
7.5 \\
6.0 \\
3.8\end{array}$ & $\begin{array}{r}9.3 \\
6.0 \\
13.5 \\
7.5 \\
7.5 \\
17.5 \\
3.8 \\
11.2 \\
7.5 \\
6.0\end{array}$ & $\begin{array}{r}11.2 \\
7.5 \\
17.5 \\
9.3 \\
11.2 \\
34.0 \\
7.5 \\
13.5 \\
11.2 \\
7.5\end{array}$ & $\begin{array}{r}11.2 \\
9.3 \\
20.0 \\
11.2 \\
13.5 \\
50.0 \\
11.2 \\
20.0 \\
17.5 \\
9.3\end{array}$ & $\begin{array}{l}13.5 \\
17.5 \\
23.0 \\
23.0 \\
17.5 \\
70.0 \\
20.0 \\
17.5 \\
34.0 \\
11.2\end{array}$ & $\begin{array}{l}17 \cdot 5 \\
34 \\
39 \\
39 \\
20 \\
84 \\
34 \\
27 \\
39 \\
13 \cdot 5\end{array}$ & $\begin{array}{c}23 \\
39 \\
50 \\
50 \\
27 \\
109 \\
70 \\
50 \\
50 \\
17.5\end{array}$ & $\begin{array}{r}39 \\
50 \\
84 \\
84 \\
34 \\
147 \\
109 \\
109 \\
109 \\
23\end{array}$ & $\begin{array}{r}109 \\
109 \\
198 \\
147 \\
50 \\
274 \\
198 \\
204 \\
198 \\
34\end{array}$ \\
\hline
\end{tabular}

Greater relative differences between the sexes are seen in case of the small weights. No woman in this group, and indeed only one in this class, selected a lead weight heavier than 3.8 grams for the equivalent of the wood disk weighing 15.5 grams. Although these results are fairly representative, it would not be safe to draw any general conclusions, regarding the relations of the factors involved, from experiments made 
upon so few persons. This table is given merely as an example of individual judgments, and it depends on the contents of the following tables for its full interpretation. The average intelligent man or woman would probably judge about as did some one of these twenty persons.

Three points are made especially prominent by Table $I$, and all are emphasized by the tables to follow. First, the women overestimate the lead nearly twice as much as do the men. Second, the over-estimation by both sexes is greatest with medium weights, and is greater for small than for large weights. Third, the amplitude of individual judgment is so great that results from many persons are necessary to establish any relation between size and weight. In this case we have to do with the element of uncertainty on the part of each observer, which may lead him to hesitate over each of, perhaps, half a dozen lead weights for a single wood disk. Add to this immense amplitude in the judgment of every individual the enormous variation between different persons, and the difficulty in obtaining exact results becomes evident.

Consider for a moment what such a judgment means. One seeks a piece of lead which feels just as heavy as the piece of wood which he holds in his hand. If the muscle sense alone be used we are able to compare weights with considerable accuracy. From 20 grams to $I, 000$ grams or more even a child, with a little practice, can recognize a difference of $\frac{1}{20}$ to $\frac{1}{100}$ of the load with some certainty. With the help of all our senses it may seem as if we ought to do better. If the materials to be compared are different in specific gravity, the introduction of other senses complicates the process, and probably will always make the results less accurate. One finds himself hesitating, therefore, not merely between two weights that seem to be about equally different from the wood in hand, but one may even be in doubt as to which of half a dozen weights is nearest like the wood; and it may be that these half-dozen leads differ not by $\frac{x}{20}$ but by 2 or even 5 times the smallest. After awhile one may eliminate some of them and come to a conclusion, but seldom is he at all certain of the correctness of results. It must be said, however, that if the right weight be offered to the ob- 
TABLE II.

Class of 1894.

\begin{tabular}{|c|c|c|c|c|c|c|c|c|c|c|c|c|c|c|c|c|c|c|c|}
\hline \multirow{2}{*}{$\frac{\text { Wood. }}{\text { MEN. }}$} & I. 25.5 & \multicolumn{2}{|c|}{$\begin{array}{l}\text { II. } \\
35 .\end{array}$} & \multicolumn{2}{|c|}{$\underset{61}{\text { III. }}$} & \multicolumn{2}{|c|}{$\begin{array}{l}\text { IV. } \\
87.5\end{array}$} & \multicolumn{2}{|c|}{$\underset{126.5}{V .}$} & \multicolumn{2}{|c|}{$\begin{array}{l}\text { VI. } \\
174\end{array}$} & \multicolumn{2}{|c|}{$\begin{array}{l}\text { VII. } \\
231\end{array}$} & \multicolumn{2}{|c|}{$\begin{array}{c}\text { VIII. } \\
295\end{array}$} & \multicolumn{2}{|c|}{$\begin{array}{l}\text { IX. } \\
3^{6} 4\end{array}$} & \multicolumn{2}{|c|}{$\underset{525}{X}$} \\
\hline & V. & A. & v. & A. & v. & $\Lambda$. & V. & A. & V. & A. & V. & A. & v. & A. & v. & A. & V. & A. & v. \\
\hline a $\left\{\begin{array}{l}6.8 \\
5.1 \\
4.8\end{array}\right.$ & $\begin{array}{r}2.1 \\
1.3 \\
.6\end{array}$ & $\begin{array}{r}13.0 \\
13.5 \\
9.5\end{array}$ & $\begin{array}{ll}5 & 1 \\
7.1 \\
1.6\end{array}$ & $\begin{array}{l}22.6 \\
21.5 \\
17.6\end{array}$ & $\begin{array}{l}9.2 \\
7.7 \\
1.6\end{array}$ & $\begin{array}{l}34.3 \\
29.6 \\
25.9\end{array}$ & $\begin{array}{r}12.6 \\
10.0 \\
3.2\end{array}$ & $\begin{array}{l}50.0 \\
40.5 \\
35.6\end{array}$ & $\begin{array}{r}13.0 \\
11.7 \\
3.7\end{array}$ & $\begin{array}{l}60.0 \\
54.7 \\
48.8\end{array}$ & $\begin{array}{r}11.9 \\
18.1 \\
5.8\end{array}$ & $\begin{array}{l}81.5 \\
77.0 \\
63.9\end{array}$ & $\begin{array}{r}18.7 \\
31.2 \\
3.3\end{array}$ & $\begin{array}{r}114.1 \\
104.3 \\
98.5\end{array}$ & $\begin{array}{l}27.7 \\
47.6 \\
29.5\end{array}$ & $\begin{array}{l}167.5 \\
153.3 \\
15^{2} .4\end{array}$ & $\begin{array}{l}47 \cdot 7 \\
63 \cdot 2 \\
43 \cdot 3\end{array}$ & $\begin{array}{l}230.2 \\
205.6 \\
229.4\end{array}$ & $\begin{array}{l}59.6 \\
60.1 \\
62.9\end{array}$ \\
\hline b $\left\{\begin{array}{l}6.6 \\
6.5 \\
5.9\end{array}\right.$ & $\begin{array}{l}1.6 \\
2.2 \\
2.2\end{array}$ & $\begin{array}{l}12.6 \\
12.1 \\
10.8\end{array}$ & $\begin{array}{l}4.3 \\
4.8 \\
4.9\end{array}$ & $\begin{array}{l}25.0 \\
23 \cdot 3 \\
189\end{array}$ & $\begin{array}{l}7.7 \\
8.1 \\
7.1\end{array}$ & $\begin{array}{l}39.9 \\
35.4 \\
30.7\end{array}$ & $\begin{array}{r}12.3 \\
9.0 \\
9.7\end{array}$ & $\begin{array}{l}53.4 \\
50.6 \\
42.7\end{array}$ & $\begin{array}{l}12.1 \\
15.0 \\
12.4\end{array}$ & $\begin{array}{l}74.8 \\
65.5 \\
53.2\end{array}$ & $\begin{array}{l}20.6 \\
181 \\
13.0\end{array}$ & $\begin{array}{r}101.4 \\
93.4 \\
73.5\end{array}$ & $\begin{array}{l}30.8 \\
26.4 \\
25.6\end{array}$ & $\begin{array}{l}132.6 \\
\mathrm{I} 20.9 \\
\mathrm{I} 33.3\end{array}$ & $\begin{array}{l}47 \cdot 4 \\
4^{2 \cdot 7} \\
4^{1 \cdot 4}\end{array}$ & $\begin{array}{l}174.0 \\
162.3 \\
166.3\end{array}$ & $\begin{array}{l}47.4 \\
59.0 \\
68.9\end{array}$ & $\begin{array}{l}276.0 \\
245.3 \\
229.4\end{array}$ & $\begin{array}{l}77.1 \\
76.1 \\
69.6\end{array}$ \\
\hline$c\left\{\begin{array}{l}7.4 \\
5.9 \\
7.8\end{array}\right.$ & $\begin{array}{l}2.8 \\
2.2 \\
3.2\end{array}$ & $\begin{array}{l}13.3 \\
12.0 \\
15.7\end{array}$ & $\begin{array}{l}5.0 \\
5.3 \\
5.8\end{array}$ & $\begin{array}{l}24.6 \\
20.6 \\
27.0\end{array}$ & $\begin{array}{r}10.8 \\
9.2 \\
10.3\end{array}$ & $\begin{array}{l}3^{8.6} \\
34.5 \\
40.3\end{array}$ & $\begin{array}{l}13.7 \\
12.3 \\
12.7\end{array}$ & $\begin{array}{l}54 \cdot 7 \\
41.8 \\
547\end{array}$ & $\begin{array}{l}18.8 \\
14.9 \\
17.6\end{array}$ & $\begin{array}{l}77.4 \\
62.2 \\
75.0\end{array}$ & $\begin{array}{l}31.9 \\
29.6 \\
295\end{array}$ & $\begin{array}{r}105.5 \\
760 \\
103.0\end{array}$ & $\begin{array}{l}52.1 \\
28.9 \\
39.6\end{array}$ & $\begin{array}{l}139.5 \\
101.4 \\
137.9\end{array}$ & $\begin{array}{l}51.0 \\
46.3 \\
40.3\end{array}$ & $\begin{array}{l}194.2 \\
163.2 \\
186.7\end{array}$ & $\begin{array}{l}69.9 \\
61.5 \\
47.9\end{array}$ & $\begin{array}{l}283.2 \\
260.1 \\
266.3\end{array}$ & $\begin{array}{l}92.4 \\
70.6 \\
75.5\end{array}$ \\
\hline $\begin{array}{l}\text { Women. } \\
\qquad\left\{\begin{array}{l}6.6 \\
5.8 \\
6.0\end{array}\right.\end{array}$ & $\begin{array}{l}1.2 \\
1.3 \\
1.8\end{array}$ & $\begin{array}{l}9.3 \\
8.3 \\
8.5\end{array}$ & $\begin{array}{l}1.8 \\
1.5 \\
2.8\end{array}$ & $\begin{array}{l}172 \\
16.4 \\
17.0\end{array}$ & $\begin{array}{l}4.6 \\
4.7 \\
4.0\end{array}$ & $\begin{array}{l}24.7 \\
22.9 \\
24.7\end{array}$ & $\begin{array}{l}7.2 \\
5.8 \\
6.2\end{array}$ & $\begin{array}{l}3^{8.6} \\
31 \cdot 1 \\
33.8\end{array}$ & $\begin{array}{r}12.5 \\
65 \\
69\end{array}$ & $\begin{array}{l}45.5 \\
42.0 \\
404\end{array}$ & $\begin{array}{r}9.2 \\
7.9 \\
10.9\end{array}$ & $\begin{array}{l}64.1 \\
52.7 \\
52.8\end{array}$ & $\begin{array}{l}15.3 \\
11.6 \\
12.1\end{array}$ & $\begin{array}{l}89.1 \\
64.5 \\
75.1\end{array}$ & $\begin{array}{r}27.5 \\
7.2 \\
26.5\end{array}$ & $\begin{array}{r}123.9 \\
82.8 \\
113.8\end{array}$ & $\begin{array}{l}48.3 \\
17.4 \\
45.8\end{array}$ & $\begin{array}{l}166.1 \\
122.9 \\
167.6\end{array}$ & $\begin{array}{l}57.7 \\
29.8 \\
83.8\end{array}$ \\
\hline e $\left\{\begin{array}{l}6.0 \\
4.9 \\
5.7\end{array}\right.$ & $\begin{array}{l}1.8 \\
1.8 \\
2.1\end{array}$ & $\begin{array}{r}9.1 \\
10.1 \\
10.1\end{array}$ & $\begin{array}{l}2.1 \\
3.0 \\
3.0\end{array}$ & $\begin{array}{l}176 \\
18.7 \\
18.1\end{array}$ & $\begin{array}{l}5.4 \\
4.9 \\
4.4\end{array}$ & $\begin{array}{l}26.6 \\
23.6 \\
24.9\end{array}$ & $\begin{array}{l}8.4 \\
3.5 \\
5.0\end{array}$ & $\begin{array}{l}34 \cdot 5 \\
33 \cdot 1 \\
347\end{array}$ & $\begin{array}{l}9.5 \\
5.0 \\
6.2\end{array}$ & $\begin{array}{l}48.7 \\
50.0 \\
44.5\end{array}$ & $\begin{array}{l}10.2 \\
147 \\
107\end{array}$ & $\begin{array}{l}66.6 \\
54.6 \\
68.9\end{array}$ & $\begin{array}{l}18.4 \\
11.7 \\
22.1\end{array}$ & $\begin{array}{l}91.0 \\
89.8 \\
84.1\end{array}$ & $\begin{array}{l}26.2 \\
34.8 \\
30.0\end{array}$ & $\begin{array}{l}123.9 \\
106.5 \\
115.7\end{array}$ & $\begin{array}{l}48 \cdot 3 \\
46 \cdot 3 \\
44 \cdot 3\end{array}$ & $\begin{array}{l}189.4 \\
164.7 \\
166.8\end{array}$ & $\begin{array}{l}56.4 \\
61.3 \\
4^{8.4}\end{array}$ \\
\hline$f\left\{\begin{array}{l}6.6 \\
5.9 \\
6.1\end{array}\right.$ & $\begin{array}{l}1.2 \\
1.9 \\
1.6\end{array}$ & $\begin{array}{l}10.8 \\
11.0 \\
11.8\end{array}$ & $\begin{array}{l}2.7 \\
4.1 \\
4.7\end{array}$ & $\begin{array}{l}20.9 \\
19.0 \\
19.5\end{array}$ & $\begin{array}{l}6.7 \\
7.7 \\
6.0\end{array}$ & $\begin{array}{l}28.1 \\
280 \\
28.3\end{array}$ & $\begin{array}{r}7.5 \\
10.6 \\
9.6\end{array}$ & $\begin{array}{l}49.7 \\
39.6 \\
39.8\end{array}$ & $\begin{array}{l}14.0 \\
11.3 \\
11.2\end{array}$ & $\begin{array}{l}65.3 \\
51.6 \\
5^{2} .3\end{array}$ & $\begin{array}{l}19.2 \\
21.6 \\
14.9\end{array}$ & $\begin{array}{l}88.3 \\
75.8 \\
69.6\end{array}$ & $\begin{array}{l}36.6 \\
37.6 \\
21.6\end{array}$ & $\begin{array}{l}125.8 \\
115.5 \\
101.4\end{array}$ & $\begin{array}{l}55.2 \\
44.4 \\
36.5\end{array}$ & $\begin{array}{r}178.2 \\
158.5 \\
\times 37.7\end{array}$ & $\begin{array}{l}88.2 \\
66.0 \\
59.7\end{array}$ & $\begin{array}{l}267.2 \\
210.9 \\
203.1\end{array}$ & $\begin{array}{r}\text { II } 6.2 \\
66.5 \\
73.9\end{array}$ \\
\hline
\end{tabular}


TABLE III.

Class of 1895.

\begin{tabular}{|c|c|c|c|c|c|c|c|c|c|c|c|c|c|c|c|c|c|c|c|}
\hline \multicolumn{2}{|c|}{ Wood. ${ }^{I .} 25.5$} & \multicolumn{2}{|c|}{$\begin{array}{l}\text { II. } \\
35\end{array}$} & \multicolumn{2}{|c|}{$\underset{6 I}{\text { III. }}$} & \multicolumn{2}{|c|}{$\begin{array}{l}\text { IV. } \\
87.5\end{array}$} & \multicolumn{2}{|c|}{$\underset{226.5}{V}$} & \multicolumn{2}{|c|}{$\begin{array}{l}\text { VI. } \\
174\end{array}$} & \multicolumn{2}{|c|}{$\begin{array}{l}\text { VII. } \\
23 \text { I }\end{array}$} & \multicolumn{2}{|c|}{$\underset{295}{\text { VIII. }}$} & \multicolumn{2}{|c|}{$\begin{array}{l}\text { IX. } \\
3^{64}\end{array}$} & \multicolumn{2}{|c|}{$\begin{array}{l}X . \\
525\end{array}$} \\
\hline MEN. & v. & A. & V. & A. & $\mathrm{V}$ & A. & V. & A. & v. & A. & V. & A. & v. & A. & v. & A. & v. & A. & v. \\
\hline $\operatorname{gg}\left\{\begin{array}{l}4.0 \\
46 \\
5.0\end{array}\right.$ & $\begin{array}{l}1.2 \\
2.1 \\
1.9\end{array}$ & $\begin{array}{l}8.0 \\
8.8 \\
8.3\end{array}$ & $\begin{array}{l}2.4 \\
2.9 \\
2.6\end{array}$ & $\begin{array}{l}13.3 \\
13.1 \\
13.6\end{array}$ & $\begin{array}{l}5.4 \\
4.6 \\
5.1\end{array}$ & $\begin{array}{l}19.0 \\
19.8 \\
18.1\end{array}$ & $\begin{array}{l}8.7 \\
6.6 \\
6.7\end{array}$ & $\begin{array}{l}30.1 \\
33.1 \\
267\end{array}$ & $\begin{array}{r}13.1 \\
13.5 \\
9.7\end{array}$ & $\begin{array}{l}41.0 \\
45 \cdot 9 \\
42 \cdot 3\end{array}$ & $\begin{array}{l}181 \\
21.7 \\
21.0\end{array}$ & $\begin{array}{l}63.5 \\
65.1 \\
65.1\end{array}$ & $\begin{array}{l}26.8 \\
31.1 \\
37 \cdot 7\end{array}$ & $\begin{array}{l}99.9 \\
90.3 \\
93.1\end{array}$ & $\begin{array}{l}413 \\
45 \cdot 4 \\
46.9\end{array}$ & $\begin{array}{l}134.6 \\
132.7 \\
131.5\end{array}$ & $\begin{array}{l}56.9 \\
551 \\
605\end{array}$ & $\begin{array}{l}204.0 \\
190.5 \\
194.6\end{array}$ & $\begin{array}{l}73.2 \\
55.0 \\
72.8\end{array}$ \\
\hline $\mathrm{h}\left\{\begin{array}{l}4.8 \\
5.6 \\
4.6\end{array}\right.$ & $\begin{array}{l}1.9 \\
2.6 \\
2.2\end{array}$ & $\begin{array}{l}10.1 \\
10.1 \\
8.9\end{array}$ & $\begin{array}{l}5 \cdot 3 \\
4 \cdot 3 \\
3.2\end{array}$ & $\begin{array}{l}19.2 \\
15.5 \\
14.4\end{array}$ & $\begin{array}{r}10.7 \\
5.5 \\
5.5\end{array}$ & $\begin{array}{l}27.4 \\
22.3 \\
20.6\end{array}$ & $\begin{array}{r}15.8 \\
8.3 \\
8.6\end{array}$ & $\begin{array}{l}39 \cdot 5 \\
34 \cdot 4 \\
31.8\end{array}$ & $\begin{array}{l}25.8 \\
15.9 \\
16.1\end{array}$ & $\begin{array}{l}58.6 \\
52.1 \\
44.2\end{array}$ & $\begin{array}{l}36.7 \\
27.2 \\
21.9\end{array}$ & $\begin{array}{l}83 \cdot 1 \\
74.8 \\
75.0\end{array}$ & $\begin{array}{l}49.0 \\
35.4 \\
43.0\end{array}$ & $\begin{array}{l}113.7 \\
1142 \\
102.4\end{array}$ & $\begin{array}{l}57.2 \\
46.7 \\
53.3\end{array}$ & $\begin{array}{l}170.1 \\
164.6 \\
145.5\end{array}$ & $\begin{array}{l}73.5 \\
570 \\
66.3\end{array}$ & $\begin{array}{l}239.9 \\
251.9 \\
235.8\end{array}$ & $\begin{array}{r}967 \\
92.9 \\
112.0\end{array}$ \\
\hline i $\left\{\begin{array}{l}3.7 \\
3.5 \\
4.1\end{array}\right.$ & $\begin{array}{r}1.0 \\
.8 \\
.9\end{array}$ & $\begin{array}{l}7.5 \\
7.7 \\
6.8\end{array}$ & $\begin{array}{l}1.3 \\
1.0 \\
1.6\end{array}$ & $\begin{array}{l}118 \\
10.8 \\
11.3\end{array}$ & $\begin{array}{l}3.2 \\
1.5 \\
2.6\end{array}$ & $\begin{array}{l}18.3 \\
151 \\
15.6\end{array}$ & $\begin{array}{l}6.2 \\
3.4 \\
4.6\end{array}$ & $\begin{array}{l}26.9 \\
20.4 \\
22.8\end{array}$ & $\begin{array}{r}10.7 \\
4.5 \\
5.8\end{array}$ & $\begin{array}{l}36.7 \\
31 \cdot 3 \\
36.4\end{array}$ & $\begin{array}{r}13.0 \\
7.0 \\
9.8\end{array}$ & $\begin{array}{l}58.1 \\
49.3 \\
58.7\end{array}$ & $\begin{array}{l}23.0 \\
16.3 \\
19.7\end{array}$ & $\begin{array}{l}794 \\
75.2 \\
88.6\end{array}$ & $\begin{array}{l}32.5 \\
28.8 \\
24.3\end{array}$ & $\begin{array}{l}121.7 \\
123.8 \\
140.0\end{array}$ & $\begin{array}{l}47 \cdot 5 \\
49 \cdot 4 \\
36.7\end{array}$ & $\begin{array}{l}199.2 \\
184.3 \\
214.6\end{array}$ & $\begin{array}{l}82.6 \\
54 \cdot 3 \\
32 \cdot 3\end{array}$ \\
\hline $\mathrm{j}\left\{\begin{array}{l}4.5 \\
5.0 \\
6.5\end{array}\right.$ & $\begin{array}{l}1.2 \\
1.6 \\
2.5\end{array}$ & $\begin{array}{r}9.1 \\
8.9 \\
12.3\end{array}$ & $\begin{array}{l}2.3 \\
2.5 \\
6.1\end{array}$ & $\begin{array}{l}13.5 \\
14.5 \\
16.6\end{array}$ & $\begin{array}{l}4.5 \\
5.9 \\
7 \cdot 4\end{array}$ & $\begin{array}{l}22.5 \\
21.0 \\
24.2\end{array}$ & $\begin{array}{r}11.1 \\
8.8 \\
10.4\end{array}$ & $\begin{array}{l}32.8 \\
29 \cdot 3 \\
34 \cdot 4\end{array}$ & $\begin{array}{l}16.4 \\
14.2 \\
15.6\end{array}$ & $\begin{array}{l}43.7 \\
41.4 \\
47.2\end{array}$ & $\begin{array}{l}18.8 \\
18.8 \\
22.5\end{array}$ & $\begin{array}{l}59.3 \\
65.0 \\
68.3\end{array}$ & $\begin{array}{l}31.8 \\
34.8 \\
38.9\end{array}$ & $\begin{array}{r}76.3 \\
108.5 \\
110.1\end{array}$ & $\begin{array}{l}31.4 \\
51.4 \\
52.5\end{array}$ & $\begin{array}{l}1175 \\
149.0 \\
167.4\end{array}$ & $\begin{array}{l}44.7 \\
76.0 \\
57 \cdot 3\end{array}$ & $\begin{array}{l}199.5 \\
215.0 \\
268.7\end{array}$ & $\begin{array}{r}90.0 \\
98.0 \\
119.3\end{array}$ \\
\hline $\begin{array}{l}\text { VOMEN. } \\
\qquad\left\{\begin{array}{l}3.4 \\
5 \cdot 3 \\
7.8\end{array}\right.\end{array}$ & $\begin{array}{l}1.2 \\
2.4 \\
5.5\end{array}$ & $\begin{array}{r}6.6 \\
9.2 \\
12.7\end{array}$ & $\begin{array}{l}2.3 \\
3.0 \\
65\end{array}$ & $\begin{array}{l}10.8 \\
15.2 \\
17.1\end{array}$ & $\begin{array}{l}4.0 \\
4.5 \\
7.9\end{array}$ & $\begin{array}{l}15.1 \\
24.0 \\
23.5\end{array}$ & $\begin{array}{r}5.4 \\
7.6 \\
11.2\end{array}$ & $\begin{array}{l}21.4 \\
32.2 \\
33.2\end{array}$ & $\begin{array}{r}8.4 \\
11.1 \\
14.4\end{array}$ & $\begin{array}{l}29.1 \\
45 \cdot 4 \\
45 \cdot 3\end{array}$ & $\begin{array}{l}16.2 \\
20.4 \\
19.4\end{array}$ & $\begin{array}{l}52.3 \\
64.7 \\
81.6\end{array}$ & $\begin{array}{l}29.9 \\
27.6 \\
34.2\end{array}$ & $\begin{array}{r}82.5 \\
94.1 \\
1035\end{array}$ & $\begin{array}{l}46.7 \\
34.7 \\
396\end{array}$ & $\begin{array}{l}101.6 \\
122.5 \\
144.5\end{array}$ & $\begin{array}{l}57.9 \\
44.9 \\
42.4\end{array}$ & $\begin{array}{l}149.1 \\
171.3 \\
204.5\end{array}$ & $\begin{array}{l}70.7 \\
5.5 \\
60.9\end{array}$ \\
\hline $1\left\{\begin{array}{l}2.8 \\
2.9 \\
3.1\end{array}\right.$ & $\begin{array}{l}.8 \\
1.0 \\
1.0\end{array}$ & $\begin{array}{l}62 \\
5.5 \\
6.0\end{array}$ & $\begin{array}{l}1.4 \\
1.0 \\
1.3\end{array}$ & $\begin{array}{l}9.7 \\
8.3 \\
8.4\end{array}$ & $\begin{array}{l}3.5 \\
1.2 \\
2.4\end{array}$ & $\begin{array}{l}13.4 \\
10.8 \\
11.0\end{array}$ & $\begin{array}{l}6.0 \\
2.2 \\
3.0\end{array}$ & $\begin{array}{l}17.1 \\
14.7 \\
14.9\end{array}$ & $\begin{array}{l}8.3 \\
3.7 \\
5.4\end{array}$ & $\begin{array}{l}23.0 \\
20.1 \\
21.8\end{array}$ & $\begin{array}{r}\text { II. } \\
7.1 \\
9.4\end{array}$ & $\begin{array}{l}3 \pi .8 \\
27.9 \\
32.6\end{array}$ & $\begin{array}{l}18.2 \\
11.1 \\
15.6\end{array}$ & $\begin{array}{l}48.0 \\
41.2 \\
49.2\end{array}$ & $\begin{array}{l}28.2 \\
21.9 \\
26.4\end{array}$ & $\begin{array}{l}66.6 \\
66.1 \\
74.5\end{array}$ & $\begin{array}{l}4^{1} \cdot 6 \\
39 \cdot 3 \\
43 \cdot 1\end{array}$ & $\begin{array}{l}126.4 \\
103.4 \\
126.7\end{array}$ & $\begin{array}{l}588 \\
48.9 \\
67.3\end{array}$ \\
\hline m $\left\{\begin{array}{l}4.2 \\
6.3 \\
4.6\end{array}\right.$ & $\begin{array}{l}2.2 \\
4.4 \\
2.2\end{array}$ & $\begin{array}{r}8.5 \\
\text { xo } 5 \\
8.4\end{array}$ & $\begin{array}{l}4.1 \\
6.1 \\
3.4\end{array}$ & $\begin{array}{l}11.7 \\
16.1 \\
14.8\end{array}$ & $\begin{array}{l}4.8 \\
9.1 \\
9.5\end{array}$ & $\begin{array}{l}16.1 \\
23.6 \\
19.9\end{array}$ & $\begin{array}{r}6.8 \\
15.1 \\
12.6\end{array}$ & $\begin{array}{l}23.2 \\
33.9 \\
26.8\end{array}$ & $\begin{array}{r}8.6 \\
18.9 \\
16.1\end{array}$ & $\begin{array}{l}34.5 \\
52.5 \\
36.0\end{array}$ & $\begin{array}{l}13.5 \\
28.0 \\
21.8\end{array}$ & $\begin{array}{l}49.1 \\
74.8 \\
56.3\end{array}$ & $\begin{array}{l}20.7 \\
35.5 \\
35.8\end{array}$ & $\begin{array}{r}78.8 \\
\text { I03.6 } \\
80.4\end{array}$ & $\begin{array}{l}37 \cdot 3 \\
50.0 \\
46.9\end{array}$ & $\begin{array}{l}105.8 \\
138.0 \\
109.5\end{array}$ & $\begin{array}{l}52.0 \\
65.5 \\
55.1\end{array}$ & $\begin{array}{l}158.2 \\
202.6 \\
157.8\end{array}$ & $\begin{array}{l}75.8 \\
91.5 \\
78.3\end{array}$ \\
\hline $11\left\{\begin{array}{l}32 \\
3.7 \\
4.8\end{array}\right.$ & $\begin{array}{r}.8 \\
.7 \\
\times .7\end{array}$ & $\begin{array}{l}6.3 \\
6.7 \\
7.7\end{array}$ & $\begin{array}{r}.9 \\
1.3 \\
2.4\end{array}$ & $\begin{array}{r}8.2 \\
9.9 \\
10.9\end{array}$ & $\begin{array}{l}1.8 \\
1.9 \\
32\end{array}$ & $\begin{array}{l}11.7 \\
14.0 \\
15.8\end{array}$ & $\begin{array}{l}2.5 \\
1.8 \\
5.4\end{array}$ & $\begin{array}{l}16.6 \\
19.0 \\
203\end{array}$ & $\begin{array}{l}5.4 \\
2.3 \\
5.8\end{array}$ & $\begin{array}{l}24.3 \\
29.7 \\
29.5 \\
\end{array}$ & $\begin{array}{l}5.8 \\
8.5 \\
8.6\end{array}$ & $\begin{array}{l}42.7 \\
39.4 \\
41.4\end{array}$ & $\begin{array}{l}12.1 \\
10.3 \\
13.6\end{array}$ & $\begin{array}{l}58.5 \\
60.4 \\
62.3\end{array}$ & $\begin{array}{l}15.5 \\
22.9 \\
24.4\end{array}$ & $\begin{array}{r}85.4 \\
103.2 \\
89.2 \\
\end{array}$ & $\begin{array}{l}21.6 \\
37.5 \\
33.8\end{array}$ & \begin{tabular}{|l}
163.0 \\
185.1 \\
142.1
\end{tabular} & $\begin{array}{l}45.3 \\
69.6 \\
45.9\end{array}$ \\
\hline
\end{tabular}


Table IV.

Classes of 1896 and 1897.

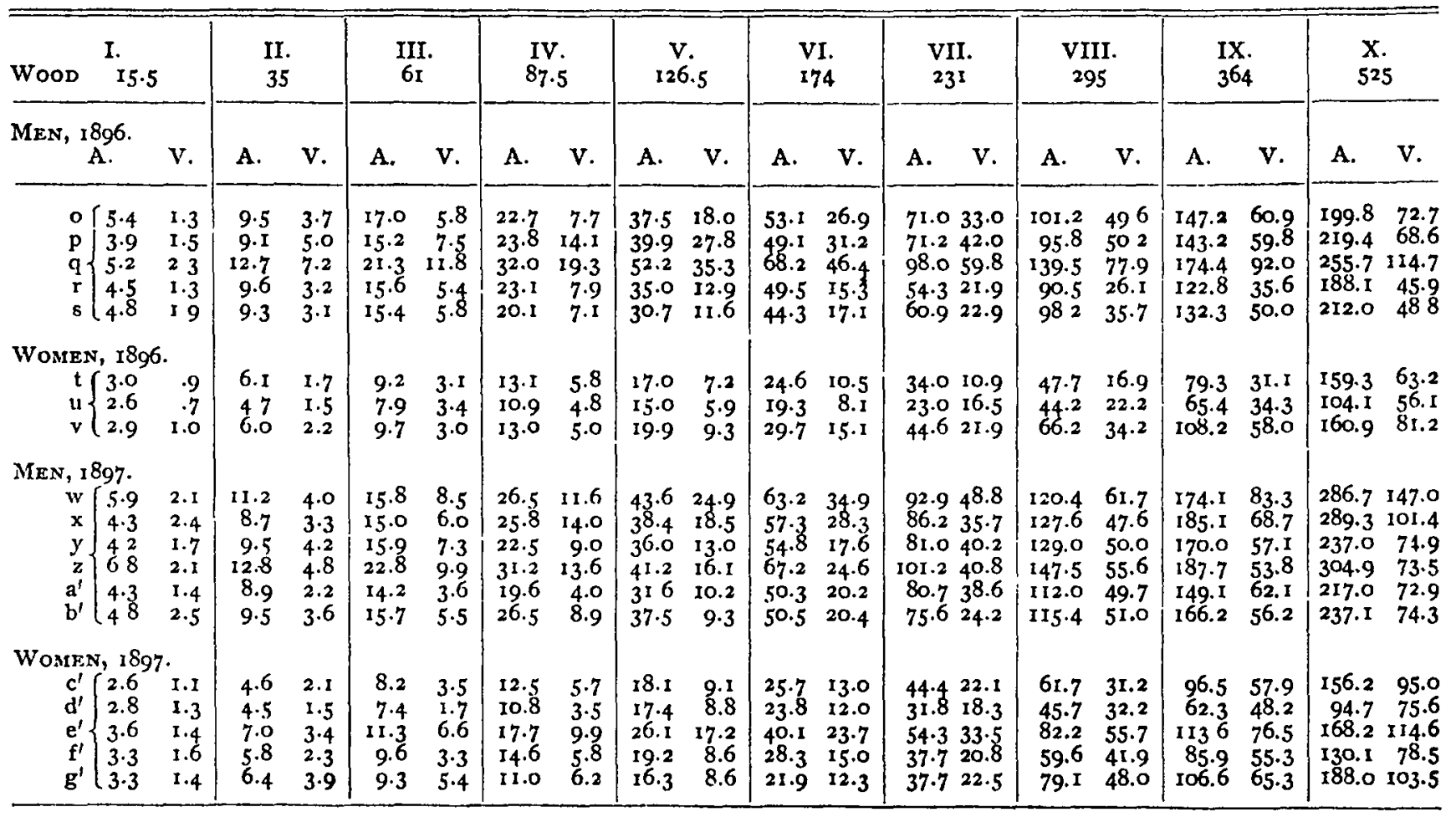


server he will reject it nine times out of ten, with every evidence of certainty in its overweight. About the only thing he is certain of is that the right weight is decidedly too heavy.

Table II. contains a summary of the results of these experiments with the students of the psychology class for the academic year '93-'94. Table III. contains the corresponding results for the class '94-'95. In each table the Roman numeral indicates the number of the wood disk, beginning with the smallest. The weight of the wood in grams is given just below. Then follow the results from the men in three divisions (groups $a, b, c$ ), and those from the women, also in three divisions (groups $d, e$, $f)$. In Table III. both men and women are divided into four divisions each.

These two classes repeated this exercise after one week, and again after another week. The average of all first judgments for each group of students is given in the first horizontal line of numbers in that group, and the average of the judgments made a week later in the second horizontal line. The average of the last judgments is recorded in the third horizontal line of each group.

Under each Roman numeral are two vertical columns of numbers. The first column contains the average weight of the lead (in grams), which seemed to be equal to the wood disk whose weight is at the head of the column. The second vertical column contains the average variation of the individuals of each group from the average of all the members of that group.

Table IV. contains the corresponding results from the classes of ' 95 -' 96 and ' $96-97$. In the former year each student made only one series, and for the latter year only the first series is given. Each group is therefore represented only by one horizontal line.

It will be observed that the average of the class of ' 93 -'94 is larger than that for any of the other classes. Especially is this true of the smaller disks. I think the difference is, perhaps, caused by the larger number of small leads provided for the later classes.

On the average the third judgment is but little greater than 
the first. This exercise was given at a time when the interest of the students was held on another part of the subject, and every precaution was taken to keep them interested in other experiments. It is possible, however, that a few students may have practiced at home and thereby may have learned something of the true relations. Yet the only person whose results seem to suggest such an explanation did not do so, I am quite certain. Of course, the students did not know they were to make another series.

Table V. contains the results from the class of ' $96-97$ at their second trial, made a week later than the first series. The wood disks were changed a little to correspond exactly with a new series of lead weights made to correct certain observed defects in those previously employed.

The lead weights increased more regularly in weight and especially in diameter and thickness. Instead of trusting to moulds for final form and size, the mould was used only for approximate size. A file was employed to cut down the disk to the desired diameter. By this means both diameter and thickness were gradually and evenly increased, retaining approximately the relation of 2 to $I$ throughout the series of leads. The weights used in this series are in grams as follows: $I, I 1 / 2,2,3$, $4,5,6,8$, I0, I2, I 5, I $8,2 \mathrm{I}, 25,3^{\circ}, 40,50,60,75$, I00, I25, I6o, $200,240,300,370,450,530,625$. This proportion has proved so satisfactory that it can safely be recommended. I would change it only for spheres or cubes in both light and heavy material.

Before attempting to account for the illusion here revealed, or to find a quantitative measure of the relations involved, let us notice some further details of these tables. The illusion is greater for women than for men. It is greater with small weights than with large weights. The variation of individuals is immense. The average variation in some of the groups amounts to more than half of the average for the group, though as a rule it runs from one-fourth to one-third in the first years, with a strong tendency to increase in later years. This is due, in part, to the better isolation of students while judging, and, in part, to the greater range in leads provided. The average vari- 
TABLE V.

Class of 7807 (New Series).

\begin{tabular}{|c|c|c|c|c|c|c|c|c|c|c|c|c|c|c|c|c|c|c|c|c|}
\hline \multirow{2}{*}{$\frac{\text { Wood. }}{\text { MEN. }}$} & \multicolumn{2}{|c|}{ I. } & \multicolumn{2}{|c|}{ II. } & \multicolumn{2}{|c|}{ III. } & \multicolumn{2}{|c|}{ IV. } & \multicolumn{2}{|c|}{ V. } & \multicolumn{2}{|c|}{ VI. } & \multicolumn{2}{|c|}{ VII. } & \multicolumn{2}{|c|}{ VIII. } & \multicolumn{2}{|c|}{ IX. } & \multicolumn{2}{|c|}{ x. } \\
\hline & A. & v. & A. & v. & A. & v. & A. & v. & A. & v. & A. & v. & A. & v. & A. & v. & A. & v. & A. & v. \\
\hline $\begin{array}{l}\mathbf{A} \\
\mathbf{B} \\
\mathbf{C} \\
\mathbf{D} \\
\mathbf{E} \\
\mathbf{F}\end{array}$ & $\begin{array}{l}4.2 \\
4.1 \\
6.1 \\
5.7 \\
3.9 \\
3.4\end{array}$ & $\begin{array}{l}1.8 \\
1.5 \\
2.3 \\
2.2 \\
1.7 \\
1.3\end{array}$ & $\begin{array}{l}8.0 \\
8.7 \\
9.9 \\
9.9 \\
6.8 \\
5.9\end{array}$ & $\begin{array}{l}3.0 \\
3.1 \\
3.3 \\
2.5 \\
3.0 \\
1.9\end{array}$ & $\begin{array}{l}14.1 \\
13.7 \\
18.5 \\
17.1 \\
11.2 \\
10.0\end{array}$ & $\begin{array}{l}6.1 \\
5.9 \\
7.1 \\
7.1 \\
4.4 \\
4.4\end{array}$ & $\begin{array}{l}25.9 \\
24.9 \\
31.0 \\
29.6 \\
18.1 \\
17.1\end{array}$ & $\begin{array}{r}11.5 \\
10.1 \\
13.4 \\
14.3 \\
6.7 \\
5.7\end{array}$ & $\begin{array}{l}35.5 \\
35.9 \\
44.1 \\
41.5 \\
25.8 \\
23.4\end{array}$ & $\begin{array}{r}18.7 \\
15.1 \\
21.7 \\
22.1 \\
10.2 \\
8.0\end{array}$ & $\begin{array}{l}53.1 \\
49.2 \\
57.0 \\
63.8 \\
37.2 \\
39.0\end{array}$ & $\begin{array}{l}17.1 \\
17.8 \\
26.0 \\
33.7 \\
15.4 \\
19.7\end{array}$ & $\begin{array}{l}78.5 \\
74.8 \\
91.0 \\
98.0 \\
591 \\
66.1\end{array}$ & $\begin{array}{l}40.9 \\
332 \\
41.0 \\
39.4 \\
23.1 \\
35.7\end{array}$ & $\begin{array}{r}115.5 \\
110.6 \\
133.0 \\
133.0 \\
93.5 \\
1044\end{array}$ & $\begin{array}{l}56.6 \\
24.2 \\
51.0 \\
51.0 \\
34.8 \\
50.4\end{array}$ & $\begin{array}{l}169.0 \\
161.0 \\
167.5 \\
179.5 \\
136.0 \\
147.2\end{array}$ & $\begin{array}{l}60.6 \\
51.6 \\
60.5 \\
76.6 \\
560 \\
78.0\end{array}$ & $\begin{array}{l}243.5 \\
238.0 \\
244.0 \\
264.5 \\
213.0 \\
241.1\end{array}$ & $\begin{array}{r}93.9 \\
68.4 \\
86.0 \\
1166 \\
79.0 \\
121.5\end{array}$ \\
\hline $\begin{array}{c}\text { WoMEN } \\
\mathbf{G} \\
\mathbf{H} \\
\mathbf{I} \\
\mathbf{J}\end{array}$ & {$\left[\begin{array}{l}3.1 \\
4.1 \\
3.6 \\
4.0 \\
3.1\end{array}\right.$} & $\begin{array}{l}1.3 \\
2.6 \\
2.0 \\
1.9 \\
1.4\end{array}$ & $\begin{array}{l}5.6 \\
8.1 \\
7.5 \\
6.8 \\
4.7\end{array}$ & $\begin{array}{l}3.1 \\
6.8 \\
4.9 \\
3.2 \\
1.6\end{array}$ & $\begin{array}{r}8.4 \\
10.9 \\
10.7 \\
10.4 \\
8.9\end{array}$ & $\begin{array}{l}4.3 \\
9.2 \\
6.2 \\
4.6 \\
3.7\end{array}$ & $\begin{array}{l}13.0 \\
15.1 \\
16.9 \\
15.4 \\
14.1\end{array}$ & $\begin{array}{r}7.4 \\
12.0 \\
8.7 \\
7.1 \\
7.1\end{array}$ & $\begin{array}{l}19.9 \\
20.1 \\
23.5 \\
21.5 \\
26.6\end{array}$ & $\begin{array}{l}10.3 \\
15.1 \\
13.2 \\
10.8 \\
18.7\end{array}$ & $\begin{array}{l}27.5 \\
26.7 \\
30.7 \\
29.3 \\
38.0\end{array}$ & $\begin{array}{l}14.0 \\
21.3 \\
17.4 \\
14.6 \\
27.8\end{array}$ & $\begin{array}{l}46.0 \\
36.9 \\
44.0 \\
42.4 \\
51.1\end{array}$ & $\begin{array}{l}25.2 \\
30.8 \\
25.0 \\
25.1 \\
36.7\end{array}$ & $\begin{array}{l}70.5 \\
58.3 \\
64.4 \\
58.9 \\
72.3\end{array}$ & $\begin{array}{l}38.6 \\
34.0 \\
40.6 \\
34.9 \\
50.8\end{array}$ & \begin{tabular}{|r}
102.1 \\
81.7 \\
89.7 \\
86.6 \\
132.4
\end{tabular} & $\begin{array}{l}47.3 \\
78.9 \\
56.3 \\
53.7 \\
70.6\end{array}$ & $\begin{array}{l}187.8 \\
109.8 \\
140.0 \\
125.0 \\
165.1\end{array}$ & $\begin{array}{r}93.0 \\
102.1 \\
84.0 \\
72.0 \\
84.9\end{array}$ \\
\hline
\end{tabular}


ation of the women is decidedly less than that of the men, though the extreme variations are, perhaps, found among the women, on account of their selection of smaller weights than any taken by the men. If it were desired to have a low variation it could easily be attained by throwing out only ro per cent. of the persons examined. We know they are 'abnormal.'

There are many small inequalities in the tables, some of which could doubtless be accounted for by reference to the larger tables from which these results are taken. Such, for example, is the case with group $k$, in which for disk I. the three successive judgments show a remarkable growth. This is explained by the fact that three of the women in that group did more thinking between times than the course of study called for. On the whole, these tables offer enough material and good enough material to warrant some general conclusions, and to control any results of another similar investigation. Certain features have come out in the progress of the work which should be carefully considered by any one else who may wish to take up a related problem.

The series of lead weights used for most of our experiments was defective, inasmuch as the diameter did not vary regularly with the weight. Only four different diameters were found in our original series of leads. The thickness of the disks increased gradually with the weight for any given diameter, yet it happened that the heaviest weight of any diameter was thicker than the lightest weight of the next larger diameter. As a rule, this fact seemed unimportant, since the lead disks were plainly numbered in the order of their weight. A few students, however, were disturbed by this sudden increase in the diameter of the disks accompanied by a decrease in thickness. The change in proportion tended to produce the same illusion as that caused by difference in specific gravity. The more compact lead disks seemed heavier than the thin disk of greater surface. Our new series of lead weights avoids this source of error by increasing both diameter and thickness as regularly and evenly as possible.

The discussion of the primary sources of the illusion is postponed till all the evidence is before us. It may be said here 
that extent of skin surface stimulated by the weights is not the principal cause of the illusion; and yet this is the explanation suggested to all who have witnessed or performed the experiments.

In a series of ten judgments such as we have in these experiments it is impossible to escape the influence of earlier judgments upon later ones. To some extent this may be observed in Table I. Such influence is very different in different individuals. For example, in numbers 7 and 8 of the men in Table $I$. the first judgments are exactly alike, but the last judgment of number 7 is four and one-half times as large as the last judgment of number 8 . On the other hand, I have noticed a few students who assumed that their first judgments were right, and then estimated that the second wood disk bore a certain relation to the first. Their self-imposed task was to find a lead disk which bore the same relation to the first lead disk. Of course, such methods were disallowed whenever suspected. We tried to obtain an independent sense-judgment in every instance.

In many other cases there was undoubtedly a strong, unconscious influence of the earlier judgments. If a different judgment had been made at the beginning of the series, it would have caused no little divergence toward the end of the series. This factor cannot be separated from the natural tendency of the individual to make about the same proportional error on each member of the series. The contrary tendency, to make each judgment without reference to previous ones, is seen in several persons. Thus it happens that a wood disk is sometimes matched with a smaller lead than was used to match a smaller wood disk just a moment before. So marked an independence in judgment is not frequent, and it is probable that the reliance on previous judgments is less potent than the personal qualities of the student.

The further question which seemed to me to be suggested by my own experience is the quantitative relation between size and apparent weight when all senses are free to act as they may. For lead and wood weights and adult persons there is no need of further experiments, except in all beginning classes in psychology, where such experiments serve to impress upon the 
minds of students the points studied in class and lecture room. Other materials both heavier and lighter, and other persons both younger and older, offer a field for interesting investigations and profitable results. It is proposed to carry this investigation into the lower grades of the public schools and to vary the weights from the lightest obtainable material to that equal in density to lead itself.

We shall now consider Table VI. with reference to the quantitative results of comparing the weights of lead and wood. It will be remembered that the results of the class of '93-' 94 are considerably different from those of the other three years, and that the apparent reason was the small number of lead weights used in the year I894. It is not probable that a further increase in the number of lead weights would much affect the judgments. $I$ have therefore brought together in Table VI. the results of the first judgments for the years ' 95 , ' 96 and ' 97 .

The first horizontal line contains the average of all the groups of men, with the average of the average variations as given for the separate groups in the preceding tables. Both of these numbers are determined directly from Tables III., IV. and $\mathrm{V}$. according to the method recommended by Fechner in case of many experiments by same observer (Psychophysik, $\mathrm{I}^{\text {to }}$ Aufl., I., 2 I $_{4}$ f.). If these groups had each included twice as many observers the average variation would have been slightly less.

This table probably gives the true relation between lead and light wood as felt by intelligent adults. We may therefore consider its facts with reference to quantitative relations between lead and wood, and also the effect on this relation of the absolute weights involved.

With the smallest wood disk we observe that the men find 4.7 grams of lead equal to 15.5 grams of wood. The women find 3.1 grams of lead equal to 15.5 grams of wood. The men think 229.2 grams of lead feel as heavy as 525 grams of wood, while the women select lead weighing only 145.2 grams as equivalent to 525 grams of wood. The entire series may be studied in the table.

We observe also the relation of wood to lead in this table. 
Table VI.

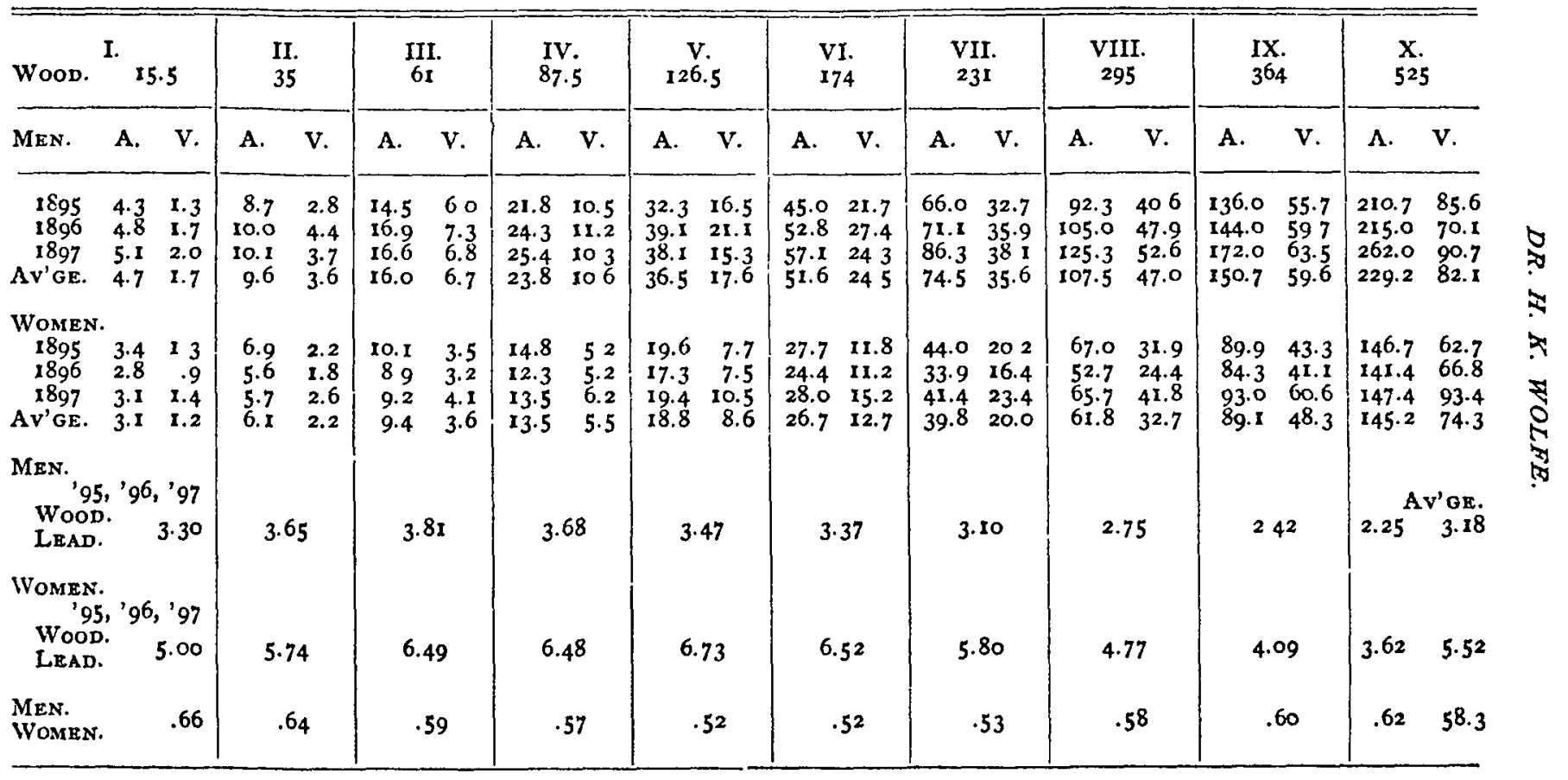


While the relation is not constant it evidently varies according to a simple law. It is not safe to say, however, that this law depends entirely upon the weight of the substances to be compared. It ought to be more generally recognized that many 'laws' of this kind have been established upon very porous foundations. It is, in fact, not improbable that a series of wood disks beginning with the size of my middle disk and extending beyond the largest of my series would show a similar law of rise and fall in the ratio of wood to lead. The constant errors arising from the order of experiments have not been eliminated in this work. The number in the series, and especially the number of lead weights at hand, probably, give rise to other constant errors.

With both men and women the ratio of wood to lead at first increases with the weight, then decreases more rapidly, till for the heaviest weight the ratio is about two-thirds as great as for the lightest weight. In case of the men the ratio increases only for the first three weights, while with the women it increases very fast for the first three weights and then more slowly for the next two, falling very little even for the sixth weight. It will be noticed that the fall in case of the men is not regularly marked until after the sixth weight is passed.

If we consider the specific gravity of the materials involved the following results are obtained. The specific gravity of the wood employed is about .45; of the lead II.35, $i$. e., the wood is to the lead as I to 25. Comparing these facts with the figures of Table VI. we find that the relations of weight and size for these materials and with these persons in the experiments already described may be formulated as follows: In equal masses the lead is twenty-five times as heavy as the wood. For equal weights the wood is twenty-five times as large as the lead. In both cases the difference is overestimated from the momentum of the contrasting sensations. The wood seems lighter and the lead heavier than it really is. If we ascribe one-half of the error to each substance, we find that, in comparing lead and wood weights, men estimate the lead at about twice its actual weight, and that they estimate the wood at about two-thirds its actual weight. Under the same conditions women estimate lead 
at more than three times its real value and wood at less than three-fifths its real value.

Of course there is no evidence in this paper that the error is due to the two substances in equal parts. The actual relation of these factors cannot be determined from my experiments. The introduction of two or three other substances for comparison with lead would furnish necessary data for a more accurate conclusion.

\section{Experiments with Paper Bags and Lead Weights.}

It is probable that the definite relation existing between weight and bulk as estimated by intelligent persons may be easily determined. It is proposed to test this question with succeeding classes. The following experiments with extreme differences in the materials indicate the need for some determination and also the apparent simplicity of the method required for the investigation.

Two light paper bags, each with a capacity of about ten litres, were obtained from a milliner. One was filled with air so that it occupied a space of perhaps $7,000 \mathrm{c} . \mathrm{cm}$. The other was loosely filled with cotton and appeared about the same in cubic contents. The first bag weighed 15.9 grams, the second weighed 106.5 grams. Each bag was suspended by a string from a fixed rod. A series of lead weights was placed on a table just below the bags; the leads beginning with .2 gram and increasing slowly but irregularly till they are connected with the series of leads used in the experiments already described.

The students worked on this problem singly. They were permitted to lift the weights as they pleased. As a rule, they lifted the paper bags by the compressed tops or by the strings, and the lead weights between the fingers or on the palm of the other hand, lifting both at the same time.

Here, as in other experiments on weights, an occasional student thought the result depended upon the manner of lifting the weights. While some little influence of this factor may be admitted, it was not observed to produce any effect as between different students. That is, the other factors are so much more powerful in determining the result that the method of lifting the 
TABLE VII.

Paper bags compared with lead weights.

\begin{tabular}{|c|c|c|c|c|c|}
\hline Mew. & LEAD. & LEAD. & MEN. & LEAD. & Lead. \\
\hline $\begin{array}{l}1 \\
2 \\
3 \\
4 \\
5 \\
6 \\
7 \\
8 \\
9 \\
10 \\
11 \\
12 \\
13 \\
14 \\
15 \\
16 \\
17 \\
18 \\
19 \\
20 \\
21 \\
22 \\
23 \\
24 \\
25\end{array}$ & $\begin{array}{r}3.8 \\
0.6 \\
6.0 \\
1.7 \\
8.8 \\
135 \\
1.7 \\
1.0 \\
11.2 \\
11.2 \\
3.8 \\
6.0 \\
2.2 \\
3.8 \\
2.2 \\
3.8 \\
3.8 \\
1.7 \\
2.2 \\
3.8 \\
3.8 \\
1.0 \\
3.8 \\
2.2 \\
0.6\end{array}$ & $\begin{array}{r}11.2 \\
50.0 \\
34.0 \\
17.5 \\
70.0 \\
50.0 \\
11.2 \\
11.2 \\
70.0 \\
147.0 \\
7.5 \\
27.0 \\
93 \\
20.0 \\
39.0 \\
50.0 \\
27.0 \\
11.2 \\
13.5 \\
50.0 \\
34.0 \\
11.2 \\
27.0 \\
34.0 \\
27.0\end{array}$ & $\begin{array}{l}26 \\
27 \\
28 \\
29 \\
30 \\
31 \\
32 \\
33 \\
34 \\
35 \\
36 \\
37 \\
38 \\
39 \\
40 \\
41 \\
42 \\
43 \\
44 \\
45 \\
46 \\
47 \\
48 \\
49 \\
50 \\
51\end{array}$ & $\begin{array}{r}11.2 \\
6.0 \\
0.6 \\
1.0 \\
11.2 \\
2.2 \\
1.0 \\
0.6 \\
3.8 \\
1.0 \\
3.8 \\
1.7 \\
2.2 \\
2.2 \\
3.8 \\
1.7 \\
3.8 \\
2.2 \\
6.0 \\
2.2 \\
9.3 \\
3.8 \\
11.2 \\
6.0 \\
11.2 \\
9.3\end{array}$ & $\begin{array}{r}70.0 \\
70.0 \\
27.0 \\
3.8 \\
39.0 \\
27.0 \\
6.0 \\
3.8 \\
17.5 \\
9.3 \\
27.0 \\
20.0 \\
39.0 \\
34.0 \\
715 \\
11.2 \\
20.0 \\
11.2 \\
20.0 \\
6.0 \\
70.0 \\
34.0 \\
50.0 \\
13.5 \\
39.0 \\
109.0\end{array}$ \\
\hline \multicolumn{3}{|l|}{ Average } & \multirow{2}{*}{\multicolumn{2}{|c|}{ WOMEN. }} & 32.26 \\
\hline WOMEN. & & & & & \\
\hline $\begin{array}{l}1 \\
2 \\
3 \\
4 \\
5 \\
6 \\
7 \\
8 \\
9 \\
10 \\
11 \\
12 \\
13 \\
14 \\
15\end{array}$ & $\begin{array}{r}1.7 \\
0.3 \\
2.2 \\
2.2 \\
0.4 \\
0.6 \\
0.3 \\
2.2 \\
1.7 \\
0.6 \\
11.2 \\
2.2 \\
1.7 \\
0.3 \\
0.3\end{array}$ & $\begin{array}{r}38 \\
7.5 \\
39.0 \\
50.0 \\
3.8 \\
13.5 \\
2.2 \\
11.2 \\
9.3 \\
3.8 \\
500 \\
20.0 \\
34.0 \\
0.6 \\
11.2\end{array}$ & $\begin{array}{l}16 \\
17 \\
18 \\
19 \\
20 \\
21 \\
22 \\
23 \\
24 \\
25 \\
26 \\
27 \\
28 \\
29 \\
30\end{array}$ & $\begin{array}{l}0.6 \\
1.7 \\
0.2 \\
3.8 \\
0.6 \\
0.4 \\
2.2 \\
6.0 \\
3.8 \\
1.0 \\
3.8 \\
1.7 \\
9.3 \\
1.7 \\
0.6\end{array}$ & $\begin{array}{r}38 \\
20.0 \\
0.6 \\
x 1.2 \\
1.7 \\
6.0 \\
13.5 \\
50.0 \\
20.0 \\
7.5 \\
23.0 \\
6.0 \\
27.0 \\
6.0 \\
6.0\end{array}$ \\
\hline Average & & & & 2.18 & 16.08 \\
\hline
\end{tabular}


weights exerted no appreciable influence. Furthermore, most of the students used several methods before finally deciding upon any lead. Table VII. gives the results of the individual judgments and the separate averages of men and women for the class of 1896 .

The averages in this table are astounding, but some of the individual judgments are nearly incredible. The reader may acquire more faith, however, by attempting to estimate the weight of an empty paste-board box in terms of lead.

The table shows that no one selected a lead weight equal to or heavier than the paper bag. In case of the smaller bag only two women and nine men selected leads half as heavy as the paper bag, while twelve women and four men selected a lead less than one twenty-fifth as heavy as the paper bag, and five women chose a lead less than one fiftieth as heavy as the bag, the average of the five being one fifty-seventh. As equivalent to the larger bag two of the women chose a lead equal to one one-hundred-and-seventy-seventh $\left(\frac{1}{1} \overline{7}\right)$ of the weight of the bag; while eighteen women and fifteen men selected leads less than one ninth as heavy as the bag, their average being, for the women, less than one eighteenth and for the men less than one twelfth.

The average of the men is for both paper bags almost exactly twice the average of the women, and this latter average is about one seventh of the true weight. It is evident from these results that the lighter the material the more difficult it is to make a comparison with the weight of lead.

It is proposed to attempt the formulation of the law expressing this relation from experiments planned for next year.

Table VIII. contains a condensed summary of results obtained from the classes of the past two years. The class of 1897 made two series of experiments. In the first series the leads of ' 96 were used. For the second series the same paper bags were employed, but the new leads of 1897 were substituted for the less regular ones of the first experiments.

For this table the students were not arranged alphabetically, as for the other tables of this paper, but they were grouped in fives, according to the weight of the lead disks selected to match 
TABLE VIII.

Paper bags compared with lead weights.

Paper bag $=\times 5.9$ Grams. $\quad$ Paptr bag $=106.5$ Grams

\begin{tabular}{|c|c|c|c|c|c|c|}
\hline MrN. & $\begin{array}{l}8 \\
8 \\
0 \\
0 \\
0 \\
0 \\
\vdots \\
3 \\
0\end{array}$ & 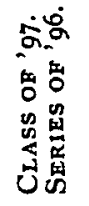 & 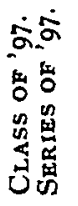 & $\begin{array}{l}0 \\
8 \\
0 \\
0 \\
0 \\
0 \\
0 \\
0\end{array}$ & 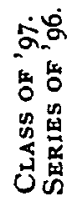 & 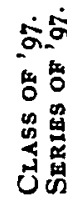 \\
\hline $\begin{array}{l}\text { Ist five. } \\
\text { 2d ““ } \\
\text { 3d “. } \\
\text { 4th “. } \\
\text { 5th “. } \\
\text { 6th “" } \\
\text { 7th ““ } \\
\text { 8th “. } \\
\text { 9th “" } \\
\text { 1oth “. } \\
\text { 1xth “ } \\
\text { 12th “ }\end{array}$ & $\begin{array}{r}.60 \\
1.00 \\
1.70 \\
2.20 \\
2.52 \\
3.80 \\
3.80 \\
4.68 \\
8.36 \\
11.20\end{array}$ & $\begin{array}{r}.78 \\
1.00 \\
1.28 \\
1.90 \\
2.20 \\
3.48 \\
3.80 \\
6.00 \\
6.00 \\
7.50 \\
8.88 \\
11.20\end{array}$ & $\begin{array}{l}.84 \\
1.10 \\
1.60 \\
2.00 \\
3.00 \\
3.00 \\
4.00 \\
4.60 \\
5.00 \\
5.80 \\
7.20 \\
9.00\end{array}$ & $\begin{array}{r}5.4 \\
97 \\
11.2 \\
16.4 \\
22.8 \\
27.0 \\
34.0 \\
41.2 \\
55.0 \\
77.8\end{array}$ & $\begin{array}{r}6.5 \\
10.1 \\
12.4 \\
15.9 \\
18.0 \\
22.4 \\
29.8 \\
34.0 \\
39.0 \\
50.0 \\
700 \\
77.0\end{array}$ & $\begin{array}{r}7.8 \\
13.4 \\
17.4 \\
18.6 \\
21.0 \\
23.4 \\
26.0 \\
300 \\
34.0 \\
46.0 \\
50.0 \\
80.0\end{array}$ \\
\hline \multicolumn{7}{|l|}{ Women. } \\
\hline $\begin{array}{l}\text { Ist five. } \\
\text { 2d “" } \\
\text { 3d “" } \\
\text { 4th “ } \\
\text { 5th “ } \\
\text { 6th “" } \\
\text { 7th “ } \\
\text { 8th “" } \\
\text { 9th “ } \\
\text { Ioth “" }\end{array}$ & $\begin{array}{r}.28 \\
.52 \\
1.12 \\
1.80 \\
2.52 \\
6.82\end{array}$ & $\begin{array}{l}.30 \\
.56 \\
.76 \\
1.00 \\
1.00 \\
1.56 \\
1.90 \\
2.52 \\
4.80 \\
6.28\end{array}$ & $\begin{array}{r}.46 \\
.84 \\
1.00 \\
1.30 \\
1.50 \\
1.70 \\
2.40 \\
3.60 \\
5.50 \\
7.50\end{array}$ & $\begin{array}{r}1.8 \\
4.7 \\
7.3 \\
12.1 \\
22.0 \\
44.6\end{array}$ & $\begin{array}{r}2.3 \\
3.8 \\
6.6 \\
9.0 \\
12.6 \\
15.1 \\
19.5 \\
23.0 \\
34.2 \\
50.0\end{array}$ & $\begin{array}{r}1.9 \\
4.0 \\
5.6 \\
8.8 \\
11.2 \\
13.8 \\
19.4 \\
26.0 \\
36.0 \\
70.0\end{array}$ \\
\hline
\end{tabular}

the paper bags. For example, the first group consists of the five men who selected the lightest lead weights as equivalent to the lightest paper bag. The average of their judgments is given in the first horizontal line. The results for the two paper bags were treated separately, hence the persons in the various groups are not the same for the two paper bags.

The only justification for such a table is lack of space to present the individual judgments. It gives a better idea of personal differences in judgments of this kind than would the aver- 
ages of large groups together with the average variations of the members of the groups. There is also less danger from freaks when an average of several persons forms the basis of conclusions.

From Table VIII. it seems that about one woman in seven finds one gram of lead equal in weight to sixty grams of inflated paper bag, and that not one woman in seven will find a gram of inflated paper bag half as heavy as a gram of lead. Men are somewhat better than women in comparing these weights, yet one man in every ten finds a gram of lead as heavy as twentyfive grams of inflated paper bag, and not one man in ten thinks a gram of inflated paper bag is three-fourths as heavy as a gram of lead.

There is little difference in the proportionate error between the two bags, but of course two members of a series would not be sufficient to determine the relations between specific gravity and apparent weight, or the effect of absolute weight upon the judgment. It is possible that the failure of a considerable difference in specific gravity to change the constant error appreciably in case of the two bags is due to some unconscious process suggested by the similar size and appearance of the bags. That it depends upon the similarity in method of raising the weight I do not believe.

We need to supplement these experiments by a series upon many persons in which the size and form of the objects remain the same, while the weight varies from the lightest possible to the specific gravity of lead itself. It is already evident that the enormous error revealed by the foregoing tables is not the effect of a simple process, but that it depends complexly upon many factors. In the conclusion of this paper I shall discuss this point more at length.

\section{Experiments with Brass Cylinders.}

As already stated the problem here studied (or a problem involving part of the same factors) was attacked in a different way by Dr. Dresslar. I thought it worth while to prepare a set of cylinders somewhat like those used by Dr. Dresslar, but with the addition of three longer and heavier cylinders. My set was 
one inch in diameter and from one to eight inches in length, increasing by half inches, except the last two of the series, which increased by one inch each. The first ten, beginning with the shortest, were all equal in weight ( 169 grams). The last three were heavier than the others, number eleven weighing 25 grams more than the first. Number twelve is 45 grams and number thirteen 75 grams heavier than the first. It will be remembered that Dr. Dresslar's cylinders were all equal in weight and in diameter, but increased regularly in length by half inches from one and one-half inches to five inches, each weighing ${ }^{1} 3^{2}$ grams.

The students were requested to arrange our cylinders in the order of their weight, beginning with the heaviest. The cylinders were placed upon a table in front of which the student sat. The suggestion was given that the weights should be lifted, one at a time, between thumb and finger. Other methods of holding the weights were not prohibited. Some students in our first year's work may have lifted a weight in each hand simultaneously, though variations from the suggested method were not numerous, and in the last two years were not permitted. Each student was given all the time he desired. The average time used was between ten and fifteen minutes. The students were not asked to express a quantitative judgment, as it is believed that psychologic method offers no place for quantitative judgments of this kind. It is true, however, that we learn, after much experience, to estimate weights in terms of an absent fixed standard, but this process is always very inaccurate, and when the standard is much less or much greater than the weight in hand the results are scarcely of scientific value. Even more difficult is it to tell how many times heavier one unfamiliar object is than another equally unfamiliar and differently constructed object. A multiple of sensations does not exist as a mental product, and when we try to attain a judgment involving such a process we do so only by referring our sensations to more or less indefinite causes of the sensations. On the other hand, equality or inequality in sensation is a plain psychologic fact.

If Dr. Dresslar had compared his shortest cylinder with lead 
weights and then his longest cylinder with the same weights, selecting such as seemed most nearly equal to each of the cylinders, he would probably have found quite a difference in the relation of these two cylinders from that given in his table. It is, in fact, observed in my series, in which the longest cylinders are actually heavier than the shortest, that the students apparently have made much smaller errors. That is, it does not seem probable that, if the shortest cylinder had produced two or three times as great sensation as the longest of those equal in weight, the effect of the greater actual weight of the last three would have become evident in the results. We notice in Table IX. that the first long cylinder which is heavier than the shortest produces an unmistakable effect on the arrangement, and that the other heavier cylinders show a corresponding increase in such effect. Now the first of the heavier weights (number II) is only 25 grams heavier than the shortest. The longest is less than fifty per cent. heavier than the shortest.

Table IX. shows the results of these experiments for the three years ' 95 , ' 96 and ' 97 . The cylinders are arranged for the table in order of their length, beginning with the shortest. The numbers at the head of the table indicate order in length. Other irregular numbers were pasted on the bottoms of the cylinders for use in recording the results after the arrangement was determined. Of course, these numbers were unknown to the student until the experiment was finished.

The first thing which attracts our attention in the table is the greater compactness in the results of the women. Further examination shows that this fact is due to the greater regularity of the women in following length as index of the weight, deciding this relation, of course, in an inverse ratio. Now it may be remembered that Dr. Dresslar concluded from his experiments that the more intelligent pupils departed less from the law than those of weaker minds. We must conclude, if this be true, that the women of these classes are brighter than the men. I shall not at present offer any further evidence on this point.

In detail the table shows, for example, that $123 \mathrm{men}$ and 107 women chose the shortest cylinder $(x)$ as the heaviest; ro men and 4 women as second heaviest; 9 men and $x$ woman as third 
TABLE IX.

Brass cylinders. Classes 1895,' 96 and ' 97 .

150 Men. 112 Women.

\begin{tabular}{|c|c|c|c|c|c|c|c|c|c|c|c|c|c|c|c|c|c|c|c|c|c|c|c|c|c|c|}
\hline Order in Length. & s & & & 2 & & 3 & & 4 & 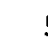 & 5 & rata & & 8 & 7 & 8 & & & 3 & & o & & I & I & & $\mathbf{I}$ & \\
\hline Sex. & $\mathbf{M}$ & $\mathbf{F}$ & $\mathbf{M}$ & $F$ & $\mathrm{~N}$ & $\mathbf{F}$ & & $\mathbf{F}$ & $\mathbf{M}$ & $\mathbf{F}$ & $\mathbf{M}$ & $\mathrm{F}$ & $\mathbf{M}$ & & $\mathbf{M}$ & & $\mathbf{M}$ & & $\mathbf{M}$ & F & $M$ & & $M$ & $\mathrm{~F}$ & $\mathrm{M}$ & $\mathbf{F}$ \\
\hline $\begin{array}{c}\text { Order in Place. } \\
1 \\
2 \\
3 \\
4 \\
5 \\
6 \\
7 \\
8 \\
9 \\
10 \\
11 \\
12 \\
13\end{array}$ & $\begin{array}{r}123 \\
10 \\
9 \\
1 \\
1 \\
1 \\
2 \\
2\end{array}$ & $\begin{array}{r}107 \\
4 \\
1\end{array}$ & $\begin{array}{r}7 \\
04 \\
18 \\
13 \\
9 \\
2 \\
3 \\
1 \\
1 \\
1 \\
1\end{array}$ & $\begin{array}{r}101 \\
6 \\
3 \\
2\end{array}$ & $\begin{array}{l}7 \\
3 \\
1 \\
1\end{array}$ & $\begin{array}{r}92 \\
\mathbf{r}_{3} \\
3 \\
2\end{array}$ & & $\begin{array}{r}4 \\
81 \\
15 \\
8 \\
3\end{array}$ & $\begin{array}{r}x \\
1 \\
2 \\
6 \\
48 \\
37 \\
26 \\
8 \\
9 \\
3 \\
3 \\
2 \\
6 \\
1\end{array}$ & $\begin{array}{r}7 \\
75 \\
13 \\
12 \\
2 \\
1 \\
2\end{array}$ & $\begin{array}{r}1 \\
5 \\
9 \\
\mathbf{3 5} \\
\mathbf{2 8} \\
25 \\
15 \\
11 \\
11 \\
5 \\
5\end{array}$ & $\begin{array}{r}1 \\
3 \\
3 \\
63 \\
21 \\
12 \\
7 \\
3 \\
1 \\
1\end{array}$ & $\begin{array}{r}2 \\
1 \\
1 \\
2 \\
13 \\
27 \\
20 \\
30 \\
29 \\
15 \\
6 \\
4\end{array}$ & $\begin{array}{r}9 \\
47 \\
13 \\
9 \\
17 \\
10 \\
2 \\
5\end{array}$ & $\begin{array}{r}1 \\
1 \\
3 \\
28 \\
21 \\
29 \\
34 \\
21 \\
15\end{array}$ & $\begin{array}{r}1 \\
1 \\
\mathbf{3 6} \\
17 \\
15 \\
12 \\
16 \\
14\end{array}$ & $\begin{array}{r}1 \\
2 \\
16 \\
10 \\
24 \\
33 \\
64\end{array}$ & $\begin{array}{l}3 \\
24 \\
10 \\
18 \\
21 \\
3^{6}\end{array}$ & $\begin{array}{r}2 \\
2 \\
2 \\
1 \\
12 \\
13 \\
21 \\
21 \\
41 \\
34\end{array}$ & $\begin{array}{r}4 \\
3 \\
27 \\
28 \\
31 \\
19\end{array}$ & $\begin{array}{r}2 \\
4 \\
10 \\
7 \\
6 \\
13 \\
13 \\
17 \\
23 \\
22 \\
22 \\
11\end{array}$ & $\begin{array}{r}1 \\
2 \\
3 \\
2 \\
15 \\
23 \\
17 \\
28 \\
16 \\
10\end{array}$ & $\begin{array}{r}1 \\
7 \\
10 \\
11 \\
12 \\
13 \\
11 \\
25 \\
20 \\
12 \\
14 \\
10 \\
4\end{array}$ & $\begin{array}{r}4 \\
1 \\
4 \\
4 \\
7 \\
14 \\
17 \\
15 \\
13 \\
10 \\
14\end{array}$ & $\begin{array}{r}17 \\
23 \\
16 \\
10 \\
20 \\
14 \\
17 \\
9 \\
5 \\
3 \\
4 \\
3 \\
9\end{array}$ & $\begin{array}{r}4 \\
7 \\
4 \\
6 \\
8 \\
9 \\
19 \\
12 \\
10 \\
6 \\
7 \\
6 \\
14\end{array}$ \\
\hline
\end{tabular}


heaviest; I man as fourth, I as fifth, I as sixth, 2 as seventh and 2 as eighth heaviest. The black-face figures show the number of men or women who placed any particular cylinder in the exact order of its size. That is, in column headed 8,23 men and $3^{6}$ women placed the eighth shortest weight in the eighth place from the apparently heaviest weight counting the latter as first place.

Observe the arrangement of those students who displaced shorter cylinders from the usual order. Of course, the shortest could be displaced only by making it come second or later. The other cylinders, however, might have been placed first or, at any rate, earlier in the series than the order of shortness required. This was very rarely done by the men and not half as often by the women.

Beginning with number ro (at top of table) there is observed a change which becomes quite marked in case of the next longer cylinder and still more so in case of the last two cylinders. The last three vertical columns show one of the most interesting facts of this whole investigation. Let us remember the continuous increase in length from $I$ to 13 and that the weight of the first ten is the same, while number II is somewhat heavier, with still further increase in $\mathbf{I} 2$ and again in $\mathbf{3} 3$. How clearly this difference has manifested itself in these results! There was no conscious reasoning in the process. The students have trusted their senses, and the factor which, I confess, had seemed to me too small to influence their judgment has very decidedly manifested itself. It shows that their discrimination is much greater than the off-hand estimates of Dr. Dresslar's school children would indicate, or perhaps it would be truer to say it shows that comparison of apparently unequal weights in terms of the lighter is exceedingly unreliable. The regularity of this curious result was entirely unsuspected until the data were tabulated in their present form at the end of the second year.

It naturally follows that the longest cylinder, which is also the heaviest, should have been most equally distributed over the entire field. This is seen to be true for both men and women. No such range of choice occurs in selecting cylinders for any particular place in the series as the horizontal lines of this table 
clearly show. The only approximation is in case of the men and with horizontal lines $6,7,8,9$, 10. Lines 7 and 8 alone contain all cylinders, but several of the places have only one representative each. This characteristic of the table is due to the fact that the shortest cylinders, without exception, were placed near the heavier end of the series. Only one of the women places any of the shortest three cylinders below sixth place, and only twenty-seven judgments of the men on these first three cylinders fall below sixth place. The cylinders of medium length are crowded down toward the lighter end of the series.

This table contains some curious facts, and it may serve as a basis for speculation. It must not be used, however, to establish quantitative relations, because the method employed cannot yield accurate results. After the shortest cylinder, the longest but one of those equal in weight, $i . e$., number 9 , offers the most interesting results. For some reason it was generally looked upon as the lightest weight, even more so than number Io, which $a$ priori consideration would have led us to expect. Number 9 was selected as the very lightest by roo students out of 262 , while number to was so regarded by only 53 students. It is not improbable that the influence of the longer and heavier cylinders is responsible for this interruption of the regular order. One of these two cylinders, however, is selected as the lightest in weight by 153 persons out of 262 , the other 109 persons distributing their judgments among 9 other cylinders, most of them falling to the three on each side of numbers 9 and to.

It is interesting to note that only men select a shorter cylinder than number 7 for the lightest, and that 38 women to 24 men select a longer cylinder than number io for the lightest. This fact is, perhaps, less indicative of female superiority than the regularity in the judgments of the women may have led us to infer.

The shortest cylinder is also the heaviest to 230 out of 262 persons. Only five women thought any other cylinder heavier. The men placed the next longer first in weight 7 times, and the longest of all the series $\mathrm{I} 7$ times.

The conflict between sight and the muscular sense is very unequal in the first part of this table. Sight evidently insists 
upon hearing the evidence and deciding the case. In the last part of the table (numbers $x 1,12,13$ ) the responsibility is pretty evenly divided.

\section{Conclusion and Summary.}

The facts stated in the preceding pages have become known, in a general way, to all persons from every-day experience. The explanations of the illusions are supposed to be the property of all thoughtful persons. Only the enormous size of the constant error is surprising. It is probable that all current theories of the cause contain a kernel of truth, but it is certain that the cause itself is exceedingly complex. Many students believed that the chief factor was the amount of surface on the hand which was stimulated. Others thought that the surface opposed to the air as the weights were lifted and balanced was most important. Some thought that the method of lifting the two kinds of weights was decisive. As a rule, the lead was lifted in the palm of the hand and the wood between the thumb and finger. Of course the students endeavored to avoid the influence of these disturbing factors whenever they were discovered.

For my part, no appreciable influence of any of these factors was observed. They doubtless have an influence, but the real cause is so much stronger than any of them that the result is not noticeably affected by varying these minor conditions. If both weights are held edgewise between the thumb and finger, the stimulated surfaces are approximately equal, the resistance of the air is reduced to a minimum, and yet the results are not perceptibly different from those obtained in the most extremely opposite manner.

It is not probable that the different temperatures of wood and lead affect our judgment of their relative weight to a noticeable extent. Whatever the value of this influence, it would, of course, tend to increase the size of the observed error.

If the weights be suspended by a cord held between the fingers, so as to give no intimation as to which is lead or wood, and if the eyes be closed and all other means of determining size be excluded, the judgment immediately improves until the error amounts to less than one-tenth of the weight; 
while with a knowledge of the relative size the error may rise to three or even ten times the weight. If the weights be held in the hands, shutting the eyes has no appreciable effect. In short, if the observer knows from any source the size of the two weights which he is comparing, he cannot avoid the influence of this factor.

It would be interesting to determine the difference between good and poor visualizers in this respect. No experiments for this purpose have been made. In fact I have not tried to discover the relative value of the numerous factors involved in the illusion now under consideration. That the visual appearance of the substances is the chief factor I am certain, though it is at least possible that the visible appearance may in turn suggest the other sensations which contribute to the general result. It seems to be the effect of space relations, and hence would not be altogether absent in experiments on the blind, provided the subjects were allowed to handle the weights as they desired.

The experiments with paper bags and those with brass cylinders contain the same factors and show similar results. It will doubtless occur to speculative minds that the cause of these illusions is the fact that the race measured gross bulk before it counted or weighed. The continued influence of this primitive method of comparison is strikingly exposed in the results of such experiments as this paper describes. To-day children and savages avoid comparison by numbers and by weight. It is not impossible that this early predilection in favor of comparison by space relations may account, in part, for the errors in judgments of weight. Of course, such an hypothesis assumes that the conflict between the early form of exclusive space comparison and the later supplementary form of comparison by weight has not yet been satisfactorily settled, and hence the novelty is still too great for the compensating pendulum of experience properly to adjust itself. The further investigation of this question should be conducted upon a few innocent persons in as many ways as possible, and for as long a time as their innocence can be maintained.

We may fairly claim that the following points have been illuminated by the present investigation: 
First. Our ordinary comparison of weights is an exceedingly complex process.

Second. Our experience with substances differing in specific gravity has not enabled us accurately to estimate their weight.

Third. Discrimination of weight decreases from $\frac{1}{20}$ of the load in similar substances to 7 times the load in substances considerably different in specific gravity (or, if we consider extreme cases, it may vary from $\frac{1}{100}$ to considerably over 100 times the standard).

Fourth. It is probable that the spatial senses of touch and sight have largely usurped the functions of the sense of weight, or else the latter is relatively a new sense and has not yet come to its full inheritance.

Fifth. The illusion of weight increases with the difference in specific gravity of the substances compared.

Sixth. Men are less prone than women to illusions of weight.

Seventh. The judgments of different persons may vary enormously, but as a rule the average of ten persons selected at random is fairly constant.

Eighth. The size of the error in case of any individual seems to be a function of his personality. It may often be foretold approximately, and is not much diminished by practice.

Ninth. Even knowledge of the true relations does not remove the error of the sense-judgment, though a person may learn from appropriate experience to make suitable allowance for the error of the senses. 